culture at the time of hospital admission in both patients and was considered to represent asymptomatic bacteriuria.

One month before identification of the first CRAB, the patients overlapped at DHMC for 8 days on different units. Potential epidemiologic links included radiology and wound care. Both patients had radiographs taken on the same day; one patient traveled to the radiology department, whereas the other had a portable radiograph. Observations of portable radiology technicians revealed consistent and adequate low-level disinfection of equipment and reliable hand hygiene. One wound-care nurse provided care to both patients on the same day. Observations of the wound-care team indicated opportunities to improve hand hygiene prior to donning and after doffing gloves; the use of single-use scissors on multiple patients; and inconsistent cleaning of a mobile device used to photograph open wounds. Discussion with the patients' outpatient providers showed that their suprapubic and wound care supplies were obtained from different companies.

Molecular analyses of the 2 patients' isolates were indistinguishable by PFGE using the restriction enzymes AscI and ApaI. Antimicrobial susceptibility testing revealed that both isolates were susceptible to colistin and resistant to all carbapenems tested. Both harbored OXA-23-like genes according to a Research Use Only assay performed at CDC.

While OXA-23-like enzymes are novel in Colorado, they were first identified in Scotland in 1985 and are the most common carbapenemase enzyme detected worldwide, accounting for $63 \%$ of nosocomial $\mathrm{CRAB}$ in Argentina, $42 \%-100 \%$ in Brazil, 98\% in Colombia, and $55 \%-80 \%$ in Saudi Arabia. ${ }^{4,6,7}$ OXA-23-like enzymes are almost exclusively found in Acinetobacter baumannii and can be encoded by genes located on either a chromosome or plasmid. ${ }^{2,5}$ OXA-23-like enzymes do not require the presence of other resistance mechanisms (eg, porin mutations or efflux pumps) to confer carbapenem resistance. However, when a bacterial strain also carries an efflux pump, the bacteria exhibit higher minimum inhibitory concentration (MIC) to carbapenems as well as resistance to multiple antibiotics, complicating the detection of the gene variant through phenotypic surveillance. ${ }^{5}$

We suspect that the organisms were transmitted during the overlapping hospital admission, although we could not determine where the organism originated or the route of transmission. On the facility level, opportunities to improve hand hygiene and lowlevel disinfection were identified and addressed. The charts were flagged to indicate that the patients harbored an MDR organism and would require contact precautions upon arrival. Infection preventionists notified clinics when upcoming outpatient appointments were detected. The clinics scheduled these patients to be the last of the clinic session when possible to allow for a thorough environmental cleaning after the clinic visit.

On a regional level, CDPHE epidemiologists contacted other healthcare facilities where these patients frequently sought care and encouraged these facilities to also electronically flag medical records and to ensure effective infection control measures. While no further cases of CRAB have been identified to date at DHMC, 1 additional OXA-23-producing CRAB case, without epidemiologic links to the previous 2 patients, has been identified in Colorado since this cluster.

The emergence of previously undetected carbapenemases in Colorado is of great public health concern. Active collaboration and communication between public health and healthcare facilities is critical to halt transmission of novel regional pathogens.

\section{Acknowledgments.}

Financial support. No financial support was provided relevant to this article.

Conflicts of interest. All authors report no conflicts of interest relevant to this article.

\section{References}

1. Munoz-Price LS, Weinstein RA. Acinetobacter infection. $N$ Engl J Med 2008;358:1271-1281.

2. Walsh TR. Clinically significant carbapenemases: an update. Curr Opin Infect Dis 2008;21:367-371.

3. Young LS, Sabel AL, Price CS. Epidemiologic, clinical, and economic evaluation of an outbreak of clonal multidrug-resistant Acinetobacter baumannii infection in a surgical intensive care unit. Infect Control Hosp Epidemiol 2007;28:1247-1254.

4. Paton R, Miles RS, Hood J, Amyes SG, Miles RS, Amyes SG. ARI 1: betalactamase-mediated imipenem resistance in Acinetobacter baumannii. Int $J$ Antimicrob Agent 1993;2:81-87.

5. Evans BA, Amyes SG. OXA beta-lactamases. Clin Microbiol Rev 2014;27:241-263.

6. Labarca JA, Salles MJ, Seas C, Guzman-Blanco M. Carbapenem resistance in Pseudomonas aeruginosa and Acinetobacter baumannii in the nosocomial setting in Latin America. Crit Rev Microbiol 2016;42:276-292.

7. Yezli S, Shibl AM, Memish ZA. The molecular basis of beta-lactamase production in gram-negative bacteria from Saudi Arabia. J Med Microbiol 2015;64:127-136.

\title{
Post-discharge impact of healthcare-associated infections in a developing country: A cohort study
}

\author{
Emília Carolina Oliveira de Souza RN, Sebastião Pires Ferreira Filho MD, MSc, Kasys Meira Gervatauskas MD and \\ Carlos Magno Castelo Branco Fortaleza MD, PhD \\ Department of Tropical Diseases, Botucatu Medical School, São Paulo State University (UNESP), City of Botucatu, São Paulo State, Brazil
}

\footnotetext{
Author for correspondence: Carlos Magno Castelo Branco Fortaleza, Departamento de Doenças Tropicais, Faculdade de Medicina de Botucatu, Distrito de Rubião Júnior, S/N, City of Botucatu, São Paulo State Brazil 18618-970. E-mail: cmfortaleza@uol.com.br

Cite this article: de Souza ECO, et al. (2018). Post-discharge impact of healthcareassociated infections in a developing country: A cohort study. Infection Control \& Hospital Epidemiology 2018, 39, 1274-1276. doi: 10.1017/ice.2018.201
}

To the Editor - The impact of healthcare-associated infections (HCAIs) on in-hospital mortality, morbidity, length-of-stay, and costs has been extensively reported. ${ }^{1,2}$ However, few studies have focused on the follow-up of HCAI-affected subjects after 

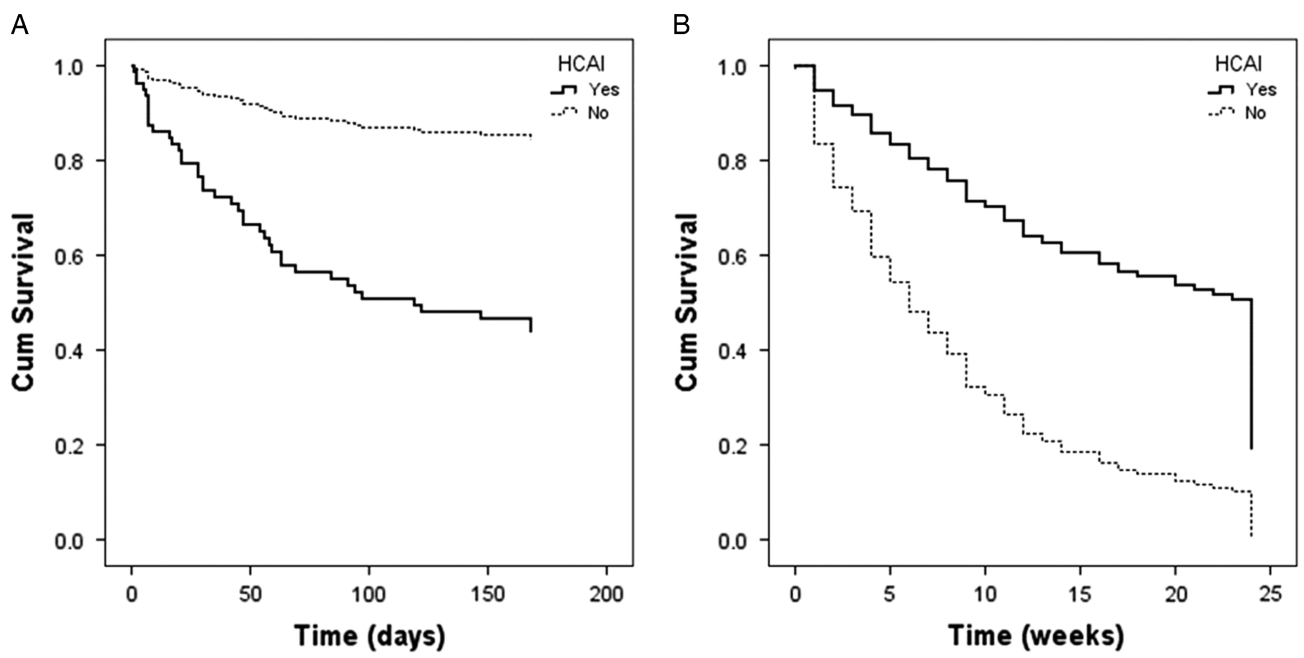

Fig. 1. Survival analysis graphics for hospital readmission and return to work or usual activities after discharge among subjects with and without healthcare-associated infections. (A) Survival graphic for hospital readmission (time counted in days). (B) Survival graphic for return to work or usual activities, with time counted in weeks. Note. $\mathrm{HCAl}$, healthcare-associated infection.

discharge. Most studies have focused on the increased risk of readmissions to hospitals. ${ }^{3-6}$ However, HCAIs may impact patient autonomy and the utilization of healthcare resources. ${ }^{7}$

We conducted a cohort study aimed at identifying the impact of HCAIs among persons discharged after diagnosis of HCAI in a teaching in inner Brazil. Adult patients discharged from Botucatu Medical School Hospital (450 beds) during 2016-2017 after diagnosis of 1 or more HCAIs were enrolled. For each subject, we included 2 controls matched by specialty (for medical patients) or by the National Healthcare Safety Network (NHSN) surgical group. ${ }^{8}$ The cohort was followed with weekly telephone calls for 24 weeks. Data recorded included (1) hospital readmissions; (2) return to work or usual daily activities (for those who did not work); (3) number of medicines taken after discharge; (4) number of medical consultations during follow up; (5) necessity of a caregiver (including family members).

Predictors of readmission and return to work or usual activities were assessed in univariate and multivariable Cox regression models. In addition to HCAIs, demographics, comorbidities (including the Charlson comorbidity index ${ }^{9}$ ), and admission data (length-of-stay, procedures, devices) were tested as predictors in those models. We used a stepwise backward strategy for selection of variables in multivariable models. $P$ values of .05 and .10 were set as limits for inclusion and exclusion of variables. Other outcomes were assessed using Mann-Whitney $U$ and $\chi^{2}$ tests, when appropriate.

We included 55 patients with HCAIs and 110 patients without HCAIs in the cohort. Among HCAI subjects, 20 had $\geq 2$ infection sites. The overall distribution of sites was as follows: surgical site infection (SSI, $\mathrm{n}=29$ ); bloodstream infection (BSI, $\mathrm{n}=20$ ); pneumonia, $(\mathrm{n}=11)$; urinary tract infection (UTI, $\mathrm{n}=9$ ); skin and soft-tissue infection (SST, $\mathrm{n}=6$ ).

Readmission during follow-up was reported for $39.3 \%$ of HCAI subjects and $18.2 \%$ of others $(P=.003)$. In our multivariable analysis, HCAI (hazard ratio [HR], 4.84; $95 \%$ confidence interval [CI], 2.20-10.63; $P<.001)$ and the Charlson comorbidity index (HR, 1.60; 95\% CI, 1.13-2.25; $P=.007)$ were significant predictors of readmission. On the other hand, HCAI was associated with later return to work or usual activities (HR, 0.30; 95\% CI, 0.19-0.57; $P<.001$ ). Other significant associations for that outcome were surgery $(\mathrm{HR}, 1.83 ; 95 \% \mathrm{CI}, 1.16-2.90 ; P=.01)$ and mechanical ventilation (HR, 0.53; 95\% CI, 0.33-0.85; $P=.009$ ). Figure 1 presents survival graphics for the impact of HCAI on readmission and return to work or usual activities. Tables with detailed results of univariate and multivariable analyzes are available as supplementary files.

The groups also differed in the following categories:

- Number of medicines taken after discharge: For HCAI, the median was 5 (quartiles [Q] 4 and 8), and for non-HCAI, the median was 4 (Q 2 and 6) $(P=.02)$.

- Number of medical consultations during follow-up: For HCAI, the median was 6 (Q 2 and 10), and for non-HCAI, the median was 3 (Q 2 and 5) $(P<.001)$.

- Number of consultations with nonmedical healthcare professionals during follow-up: For HCAI, the median was 1 (Q 0 and 2), and for non-HCAI, the median was 0 (Q 0 and 1) $(P=003)$.

Finally, $20.0 \%$ of subjects in the HCAI group required that a family member quit work (either definitively of temporarily) to be a caregiver, a situation reported by only 1 subject $(0.9 \%)$ in the non-HCAI group $(P<.001)$.

Our results agree with those of previous studies. Sreeramoju et $\mathrm{al}^{3}$ identified HCAIs as a direct cause of hospital readmissions, while Emerson et $\mathrm{al}^{4}$ (studying a very large retrospective cohort) found that subjects with infections caused by methicillin-resistant Staphylococcus aureus (MRSA), vancomycin-resistant enterococci (VRE), and Clostridium difficile were more likely to be readmitted. Schor et $\mathrm{al}^{5}$ reported that patients discharged after treating healthcare-associated pneumonia were 7.5 times more likely to be readmitted within 30 days of discharge than those treated for community-acquired pneumonia. Finally, Gohil et al ${ }^{6}$ identified higher rates of infection-related readmissions among hospitals caring for populations with higher comorbidity and poverty rates.

However, our focus went beyond readmissions. We used return to work or usual activities as a proxy for patient autonomy and found that HCAIs had a significantly negative impact on that outcome. Other findings (eg, greater use of medicines and the number of medical and nonmedical consultations) were 
consistent with results from a study of the postdischarge impact of MRSA. ${ }^{7}$

Our study was limited by the small number of subjects and the relatively short follow-up period. Also, we did not perform analysis of postdischarge healthcare costs of HCAI. However, our study also has strengths: the prospective design, the analysis of several relevant outcomes and the focus on all sites of infection. To our knowledge, this is the first study of postdischarge impact of HCAI conducted in a developing country. These countries face the paradox of having a greater burden of HCAI, and they have fewer resources to provide care to affected patients. ${ }^{10}$

In conclusion, HCAI impacted hospital readmissions, later return to work or usual activities, greater use of medicines, and number of medical consultations. These are challenging areas for developing countries and reinforce the importance of including HCAI in the public health agenda.

Supplementary materials. To view supplementary material for this article, please visit https://doi.org/10.1017/ice.2018.201

Acknowledgments. All authors state that they have no conflict of interest regarding this study.

Financial support. C.M.C.B.F. received a researcher grant from the National Council for Scientific and Technological Development (CNPq, grant no. 312149/2015-8), Brazil. K.M.G. participated part of this study as a medical student, with a grant from the São Paulo Research Foundation (FAPESP, grant no. 2014/04498-6), with C.M.C.B.F as advisor.

Conflicts of interest. All authors report no conflicts of interest relevant to this article.

\section{References}

1. Umscheid CA, Mitchell MD, Doshi JA, Agarwal R, Williams K, Brennan PJ. Estimating the proportion of healthcare-associated infections that are reasonably preventable and the related mortality and costs. Infect Control Hosp Epidemiol 2011;32:101-114.

2. Barrasa-Villar JI, Aibar-Remón C, Prieto-Andrés P, Mareca-Doñate R, Moliner-Lahoz J. Impact on morbidity, mortality, and length of stay of hospital-acquired infections by resistant microorganisms. Clin Infect Dis 2017;65:644-652.

3. Sreeramoju P, Montie B, Ramirez AM, Ayeni A. Healthcare-associated infection: a significant cause of hospital readmission. Infect Control Hosp Epidemiol 2010;31:1195-1197.

4. Emerson CB, Eyzaguirre LM, Albrecht JS, Comer AC, Harris AD, Furuno JP. Healthcare-associated infection and hospital readmission. Infect Control Hosp Epidemiol 2012;33:539-544.

5. Shorr AF, Zilberberg MD, Reichley R, et al. Readmission following hospitalization for pneumonia: the impact of pneumonia type and its implication for hospitals. Clin Infect Dis 2013; 57:362-367.

6. Gohil SK, Datta R, Cao C, et al. Impact of hospital population case-mix, including poverty, on hospital all-cause and infection-related 30-day readmission rates. Clin Infect Dis 2015;61:1235-1243.

7. Nelson RE, Jones M, Liu CF, et al. The impact of healthcare-associated methicillin-resistant Staphylococcus aureus infections on post-discharge healthcare costs and utilization. Infect Control Hosp Epidemiol 2015;36: 534-542.

8. National Healthcare Safety Network. Surgical site infection (SSI) event. Atlanta, GA: Centers for Disease Control and Prevention, 2018.

9. Charlson ME, Pompei P, Ales KL, MacKenzie CR. A new method of classifying prognostic comorbidity in longitudinal studies: development and validation. J Chronic Dis 1987; 40:373-383.

10. Allegranzi B, Bagheri Nejad S, Combescure C, et al. Burden of endemic health-care-associated infection in developing countries: systematic review and meta-analysis. Lancet 2011;377:228-241. 